OPEN ACCESS

Edited by:

Umberto Malapelle,

University of Naples Federico II, Italy

Reviewed by:

Yuchen Liu,

Shenzhen University, China

Francesco Pepe

University of Naples Federico II, Italy

${ }^{*}$ Correspondence:

Kuiran Liu

liukr@sj-hospital.org

Specialty section: This article was submitted to

Women's Cancer,

a section of the journal

Frontiers in Oncology

Received: 17 October 2020 Accepted: 29 December 2020

Published: 18 February 2021

Citation:

Jiang L, Wan Y, Feng Z, Liu D, Ouyang L, Li Y and Liu K (2021) Long Noncoding RNA UCA1 Is Related to

Autophagy and Apoptosis in

Endometrial Stromal Cells.

Front. Oncol. 10:618472.

doi: 10.3389/fonc.2020.618472

\section{Long Noncoding RNA UCA1 Is Related to Autophagy and Apoptosis in Endometrial Stromal Cells}

\author{
Lili Jiang ${ }^{1}$, Yahui Wan ${ }^{1}$, Ziyi Feng ${ }^{2}$, Da Liu ${ }^{1}$, Ling Ouyang ${ }^{1}$, Yan Li ${ }^{1}$ and Kuiran Liu ${ }^{1 *}$ \\ ${ }^{1}$ Department of Obstetrics and Gynecology, Shengjing Hospital of China Medical University, Shenyang, China, ${ }^{2}$ College of \\ Clinical Medicine Science, China Medical University, Shenyang, China
}

Research Question: The expression of the long noncoding RNA (IncRNA) urothelial carcinoma-associated 1 (UCA1) in embryonic tissues is higher than that in most cancer tissues, such as bladder cancer, indicating that RNA is a carcinoembryonic antigen. However, there are no published reports on the role of UCA1 in endometriosis (EMS). Therefore, to address this gap in knowledge, we assessed the potential role of IncRNA UCA1 in the pathogenesis and progression of EMS.

Design: To verify the expression of UCA1 in EMS, quantitative reverse transcription polymerase chain reaction (qRT-PCR) was used. RNA interference (siRNA) was used to study the biological function of UCA1 in EMS in vitro.

Results: qRT-PCR analysis showed that the expression of IncRNA UCA1 in EMS was increased $(P<0.01)$. Knockdown of UCA1 in vitro significantly inhibited the proliferation of endometrial stromal cells (ESCs) and induced autophagy and apoptosis.

Conclusion: UCA1 is highly expressed in EMS and promotes the proliferation of ESCs but suppresses autophagy and apoptosis. In EMS, UCA1 may be a prognostic marker and therapeutic target.

Keywords: urothelial carcinoma-associated 1, endometriosis, autophagy, apoptosis, light chain 3, vacuole membrane protein 1

\section{INTRODUCTION}

Endometriosis (EMS) is a common benign gynecological disease that is estrogen-dependent. The growth and proliferation of endometrial tissues or cells outside the uterus, most commonly in the ovaries and pelvic peritoneum, are characteristic of EMS. In reproductive age, $10 \%-15 \%$ women are affected by endometriosis. Under the influence of the disease, $70 \%$ of the patients suffer from chronic pelvic pain and $48 \%$ have fertility problems (1). The main clinical complications of this disease include severe menstrual, several nonmenstrual pain and subfertility (1). The most generally acknowledged hypothesis about the pathogenesis of EMS is the implantation and growth of disseminated endometrial fragments and viable cells after retrograde flow of menses during menstruation (2). Although menstrual reflux is accepted, it does explain why $90 \%$ of women experience menstrual retrograde but that only $10 \%$ develop the disease (3). The pathogenesis of 
EMS remains uncertain. The identification of novel treatments and new prognostic indicators is needed.

Long noncoding RNAs (lncRNAs) are more than 200 nucleotides long and do not have protein-coding functions but participate in significant biological activities (4). Research on lncRNAs has initially revealed the etiology and pathogenesis of certain tumors or other diseases. At present, more than ten lncRNAs related to autophagy have been found. However, the biological role of lncRNAs in EMS remains unclear.

As a type of IncRNA, urothelial carcinoma-associated 1 (UCA1) was first identified in bladder transitional cell carcinoma (5). UCA1 is highly expressed in numerous cancers, including non-small cell lung cancer, gastric cancer and ovarian cancer. UCA1 was found to be a very sensitive and specific tumor-specific marker. It can play an important role in tumor diagnosis, treatment, prognosis, and postoperative non-invasive follow-up, suggesting that UCA1 may play a key role in human cancers or other diseases (6-9).

Endometriosis is still a major challenge for reproductive health due to multiple causes, coupled with the heterogeneity of the disease and the lack of appropriate diagnostic markers and treatment methods (10). Previous studies have shown that EMs is associated with changes in the expression of long non-coding RNAs (lncRNAs). Yu et al. (11) have found that lncRNA MALAT1 can promote endometrial cell apoptosis and regulate the expression of MMP-9 through the NF- $\mathrm{KB} / \mathrm{iNOS}$ pathway, thereby mediating the pathogenesis of EMs. Other studies have found that LncRNA BANCR can inhibit the development of ectopic endometrium by repressing the formation of angiogenesis factors in EMs (12). It is concluded that lncRNAs is related to the occurrence and development of EMs, and may play a key role in prognostic, diagnostic or therapeutic role in EMs. In this study, we discovered that UCA1 was overexpressed in EMS. We studied the effect of UCA1 on the proliferation, autophagy and apoptosis of ESCs in vitro. This is the rare report investigating the role of UCA1 in EMS.

\section{MATERIALS AND METHODS}

\section{Tissue Collection}

All patients were from Shengjing Hospital, China Medical University. First, 30 surgical patients (23-51 years old) with EMS and 30 surgical patients $(22-50$ years old) with cervical intraepithelial neoplasia (CIN) were selected to obtain endometrial tissue specimens. There was no significant difference in pregnancy, birth or age between the two groups. None of these patients received any hormonal drugs or $\mathrm{GnRH}$ analog within the 6 months before the surgery. The patients didn't implant intrauterine devices. Those with other diseases of the reproductive system, as well as those with severe organ failure, malignant tumors or complications were excluded. The clinical characteristics of the patients of the two groups shown in Table 1. Fresh, sterile specimens were frozen in liquid nitrogen and stored at $-80^{\circ} \mathrm{C}$. After our conclusion was in line with the forecast, fifteen patients (28-47 years old) without birth requirements with stage III-IV ovarian EMS (according to the revised American Fertility Society classification system) undergoing total hysterectomy were selected for the subsequent study. All diagnoses were pathologically confirmed. The collection of specimens was authorized by the patient herself or her family. The specimens collected for use in this study were approved by the China Medical University Research Ethics Committee.

\section{Cell Isolation and Culture}

Endometrial stromal cell (ESC) cultures were isolated from endometrial tissues according to the following protocol. In brief, endometrial tissue samples were cut into small pieces and digested in $0.1 \%$ collagenase (type I) (Sigma-Aldrich, Shanghai, China) for 60 min with a shaking water bath at $37^{\circ} \mathrm{C}$. The digested cells were passed through a nylon sieve (aperture of $38 \mu \mathrm{m}$ ), and the eluate containing the stromal cells was then centrifuged at $1000 \mathrm{r}$ for $10 \mathrm{~min}$ at room temperature. The obtained cell pellet was washed once and resuspended in DMEM/F12 (HyClone, Logan, Utah, USA) supplemented with $10 \%$ fetal bovine serum (FBS; Gemini, Calabasas; CA, USA) in a sterile incubator at $37^{\circ} \mathrm{C}$ and $5 \% \mathrm{CO}_{2}$. The purity of the cell cultures was higher than $99 \%$ after the third passage as determined by analysis of cellular markers via immunocytochemical staining methods (all antibodies were from Zhongshan Goldenbridge, Beijing, China). Cells from passage 3 to passage 5 were used in these experiments.

\section{Isolation of Total RNA, Reverse Transcription, and Quantitative Reverse Transcription Polymerase Chain Reaction}

TRIzol reagent (9108, Invitrogen, Carlsbad, CA, USA) was used to isolate total RNA from tissues and cells. All steps were performed without RNase. RNA optical density (OD) and concentration were read by a UV spectrophotometer (Bio-Rad, California, USA). A PrimeScript RT reagent kit (RR047A, TaKaRa Bio Inc., Japan) was used to perform the reverse transcription reactions. A SYBR PremixEx TaKaRa kit (RR420A, TaKaRa Bio Inc., Japan) and an

TABLE 1 | Clinical characteristics of the patients in EMS group and normal endometrium group.

\begin{tabular}{|c|c|c|c|c|}
\hline Characteristics & EMs group $(n=30)$ & Normal endometrium group $(n=30)$ & $t / \chi^{2}$ & P Value \\
\hline Age & $36.63 \pm 7.87$ & $38.87 \pm 7.96$ & -1.093 & 0.279 \\
\hline Gravidity & $2.06 \pm 0.89$ & $2.49 \pm 1.09$ & -1.365 & 0.178 \\
\hline Parity & $1.20 \pm 0.61$ & $1.53 \pm 0.78$ & -1.849 & 0.07 \\
\hline \multicolumn{5}{|l|}{ Menstruation } \\
\hline None-Menopause & 28 & 26 & 0.741 & 0.389 \\
\hline Menopause & 2 & 4 & & \\
\hline
\end{tabular}


ABI 7500 fast real-time system (Life Technologies, Foster City, CA, USA) were used for qRT-PCR. All steps of qRT-PCR strictly followed the manufacturers' protocols.

Relative lncRNA mRNA expression levels (mean \pm SD) were calculated using the $2^{-\triangle \triangle \mathrm{Ct}}$ method with glyceraldehyde-3phosphate dehydrogenase (GAPDH) as the endogenous control. Each sample was assayed in triplicate. The qRT-PCR primers were designed by Primer Premier 5.0 software (Premier, Canada), and their sequences are shown in Table 2.

TRIzol reagent (9108, Invitrogen, Carlsbad, CA, USA) was used to isolate total RNA from tissues and cells. All steps were performed without RNase. RNA optical density (OD) and concentration were read by a UV spectrophotometer (Bio-Rad, California, USA). One microgram of RNA was reverse transcribed using the Prime Script RT reagent kit (RR047A, TaKaRa Bio Inc., Japan). The QuantiTect SYBR Green RT-PCR Kit (QIAGEN) was used to prepare the qPCR reaction. PCRs were performed on an ABI PRISM 7500 sequence detection system (Applied Biosystems; Thermo Fisher Scientific, Inc.). For the measurement of miRNA expression, miRNA-specific cDNA was synthesized from $5 \mathrm{ng}$ of total RNA using the TaqMan MicroRNA Reverse Transcription Kit (Applied Biosystems) on an ABI PRISM 7500 sequence detection system (Applied Biosystems). The expression levels of U6 small nuclear RNA and $\beta$-actin mRNA were used as reference genes. All PCRs were repeated 3 times, and the mean values are presented. Fold changes in gene expression were measured using the $2^{-\Delta \Delta C T}$ method. The primer sequences are shown in Table 2.

\section{Lentiviral Transfection}

LncRNA UCA1 siRNA (si-UCA1) and a negative control (siRNA-NC) were purchased from GeneChem (Shanghai, China). The sequences of these siRNAs are shown in Table 3. Both siRNAs were transfected into cells cultured in 6-well plates following the manufacturer's instructions. Our study had the following two controls: 1) cells transfected with siRNA negative control (siRNA-NC); and 2) cells with no siRNA transfection, which was also called the mock control (Control). As recommended by the manufacturer's instructions, the knockdown effect of si-UCA1 was assayed by qRT-PCR after transfection for $72 \mathrm{~h}$. Every patient had individual cultures.

\section{Cell Proliferation Assay}

Cell proliferation was measured using the Cell Counting Kit-8 assay (CCK-8; Dojindo Molecular Technologies, Osaka, Japan). Briefly, ESCs were seeded into 96-well plates and transfected with siRNA. After culturing for $0,24,48$, and $72 \mathrm{~h}, 10 \mu \mathrm{l}$ of CCK-8 reagent was added to all wells at $37^{\circ} \mathrm{C}$ for $1 \mathrm{~h}$. Cells were then incubated for an additional $4 \mathrm{~h}$. The absorbance at $450 \mathrm{~nm}$ of each well was measured using a microplate reader (Bio-Rad, Hercules, CA, USA). The cell proliferation rate was calculated by the ratio of the OD values of the experimental and control groups.

\section{Cell Lysosomal Fluorescence Intensity Assay}

Cell lysosomal fluorescence intensity was estimated with a LysoTracker Red kit (Beyotime Biotechnology, Beijing, China).
TABLE 2 | Primer sequences used in qRT-PCR.

\begin{tabular}{ll}
\hline RNA & \multicolumn{1}{c}{ Primers } \\
\hline UCA1 & Forward: 5'-TTCCACATATTGGCAACCAGAC-3' \\
& Reverse: 5'-GATTAAGCTGAGGCTGGCAAAG-3' \\
LC3 & Forward: 5'-AACATGAGCGAGTTGGTCAAG-3' \\
& Reverse: 5'-GCTCGTAGATGTCCGCGAT-3' \\
VMP1 & Forward: 5'- TATGCCAAACGAATCCAGCAG -3' \\
& Reverse: 5'- CCAGTCTGTTGCAAGTTGCTG -3' \\
GAPDH & Forward: 5'-GCACCGTCAAGGCTGAGAAC-3' \\
& Reverse: 5'-ATGGTGGTGAAGACGCCAGT-3' \\
\hline
\end{tabular}

TABLE 3 | siRNA sequences.

SiRNA

Target Seq

Si-UCA1

SiRNA-NC

CTCCTGGAAGCCACAAGATTA TTCTCCGAACGTGTCACGT

Higher fluorescence intensity represents stronger lysosomal activity. LysoTracker Red was added to each well, and the plates were incubated at $37^{\circ} \mathrm{C}$ for $2 \mathrm{~h}$ according to the manufacturer's protocol. LysoTracker Red was then removed, and fresh medium was added. A fluorescence microscope (Nikon Eclipse NI microscope, Nikon, Inc., Japan) was used for imaging. Lysosomes showed bright fluorescence staining, and the results were analyzed by ImageJ analysis software.

\section{Cell Apoptosis Assay}

An annexin V FITC apoptosis detection kit (Annexin FITC, Dojindo Molecular Technologies, Osaka, Japan kit) was used to analyze cell apoptosis. Cells were washed with $1 \mathrm{x}$ PBS and then diluted to $1 \times 10^{6}$ cells $/ \mathrm{ml}$ in $1 \times$ binding buffer. Subsequently, 10 $\mu \mathrm{l}$ of annexin V and PI (1:1) was added to $100 \mu \mathrm{l}$ of the cell suspension. After incubation for an additional $15 \mathrm{~min}$ in the dark, $400 \mu \mathrm{l}$ of $1 \times$ binding buffer was added to the media. FACS Calibur (Becton, Dickinson and Company, Franklin Lake, New Jersey, USA) analysis was used to detect and quantify positive cells.

\section{Western Blot Assay}

Western blotting was performed as previously described (13). Anti-VMP1 was purchased from Abcam (Cambridge, Massachusetts, USA). Anti-LC3, anti-GAPDH and anti-cleaved PARP were purchased from ProteinTech Group (Wuhan, China). GAPDH was used as the control. The individual LC3 and VMP1 band intensities were then normalized to the corresponding GAPDH absorbance value. The band intensity was visualized and analyzed by an imager (Bio-Rad, Shanghai, China) after digitization with a scanner (Bio-Rad, CA, USA). The optical densities of the protein bands were measured using Image Lab software (Bio-Rad, Shanghai, China) after digitization with a scanner (Bio-Rad, CA, USA). GAPDH was used as the internal control. The band intensities of LC3 and VMP1 were then normalized to the corresponding GAPDH absorbance value.

\section{Statistical Analyses}

All data was expressed as the mean \pm SEM. SPSS 17.0 software (SSPS Inc., Chicago, USA) was used for the statistical tests. 
Statistical comparisons of means between groups were performed using paired Student's t-test or ANOVA (one-way) followed by Tukey's test. $\mathrm{P}<0.05$ was considered statistically significant.

\section{RESULTS}

\section{Expression of IncRNA UCA1 in EMS Tissue}

qRT-PCR revealed that the expression of IncRNA UCA1 was significantly higher in the eutopic endometrium of patients with endometriosis $(0.965 \pm 0.105)$ than in normal endometrial tissues $(0.196 \pm 0.079)(\mathrm{P}<0.01$; Figure 1A).

\section{Interference Effect of UCA1 siRNA in ESCs}

After $72 \mathrm{~h}$ of siRNA transfection, the expression of UCA1 in ESCs was detected by qRT-PCR. Compared with control cells and siRNA-NC-transfected cells, UCA1 expression in ESCs transfected with si-UCA1 was decreased $(\mathrm{P}<0.01$; Figure 1B).

\section{Effect of UCA1 on the Proliferation of ESCs In Vitro}

The CCK- 8 assay was used to determine whether lncRNA UCA1 affects the proliferation of ESCs in vitro. Compared with siRNA NC-transfected cells and control cells, the proliferation of ESCs decreased significantly after si-UCA1 transfection $(\mathrm{P}<0.05$; Figure 1C; Table 4).

\section{Effect of UCA1 on the Lysosomal Fluorescence Intensity of ESCs In Vitro}

A fluorescent staining assay was used to determine whether IncRNA UCA1 affects the lysosomal activity of ESCs in vitro. Compared with the control and siRNA-NC groups, the lysosomal fluorescence intensity of ESCs was significantly higher after transfection with si-UCA1 $(\mathrm{P}<0.05$; Figures 2A, B).

\section{Effect of UCA1 on ESC Apoptosis In Vitro}

The FACS assay was used to determine whether lncRNA UCA1 affects the apoptosis of ESCs in vitro. Compared with control and

\section{theng}
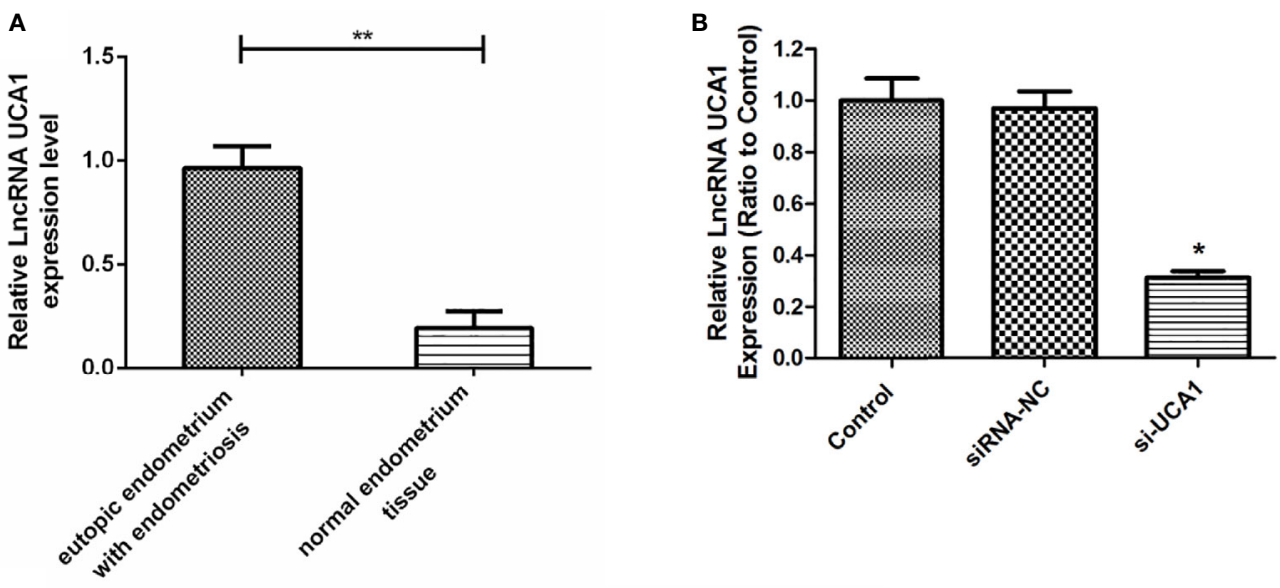

c

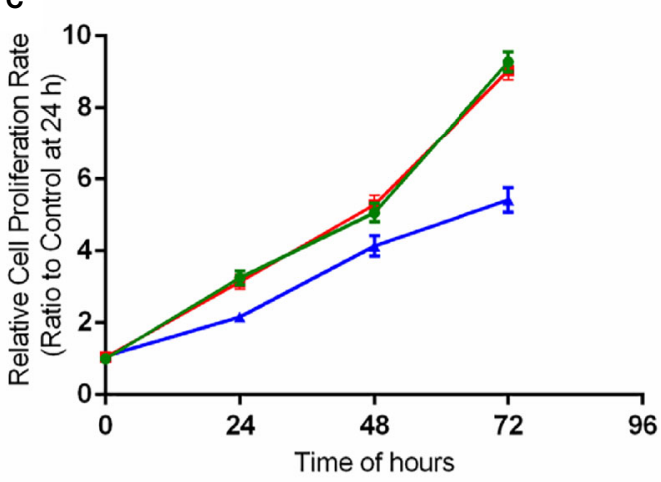

$\rightarrow$ Control

$\rightarrow$ siRNA-NC

$\mp$ si-UCA1

FIGURE 1 | Expression of IncRNA UCA1 in EMS tissue. (A) The expression level of IncRNA UCA1 in the eutopic endometrium of patients with endometriosis was higher than that in normal endometrial tissues by qRT-PCR ( $\left.{ }^{*} \mathrm{P}<0.01\right)$. The interference effect of si-UCA1in ESCs. (B) Compared with the control and siRNA-NC groups, the expression levels of UCA1 in ESCs transfected with si-UCA1 were significantly decreased ("P<0.05). Effect of UCA1 on the proliferation of ESCs in vitro. (C) The proliferation abilities of cells decreased significantly after transfection with si-UCA1 compared to the siRNA-NC and control groups ( ${ }^{*}<<0.05$, $\left.{ }^{*} \mathrm{P}<0.01\right)$. 
TABLE 4 | Changes in cell proliferation after transfection with si-UCA1 compared with siRNA-NC and control cells.

\begin{tabular}{|c|c|c|c|c|c|}
\hline Times & Control & SiRNA-NC & si-UCA1 & P Value (Control vs. siUCA1) & P Value (Control vs. siRNA-NC) \\
\hline $\mathrm{Oh}$ & $1.00 \pm 0.09$ & $1.05 \pm 0.09$ & $1.08 \pm 0.09$ & - & - \\
\hline $24 \mathrm{~h}$ & $3.25 \pm 0.19$ & $3.14 \pm 0.21$ & $2.16 \pm 0.12$ & $0.001^{\star}$ & 0.6964 \\
\hline $48 \mathrm{~h}$ & $5.06 \pm 0.26$ & $5.29 \pm 0.26$ & $4.14 \pm 0.28$ & $0.001^{*}$ & 0.5343 \\
\hline $72 \mathrm{~h}$ & $9.28 \pm 0.27$ & $9.04 \pm 0.25$ & $5.42 \pm 0.34$ & $0.001^{*}$ & 0.5263 \\
\hline
\end{tabular}

siRNA-NC-transfected cells, the apoptosis rate of ESCs was significantly increased after si-UCA 1 transfection $(\mathrm{P}<0.05$; Figures 2C, D).

\section{Effect of UCA1 on LC3 and VMP1 Expression}

LC3 is a marker protein on the autophagosome membrane. Since LC3 is needed during the formation of the autophagosome membrane, the autophagosome can be identified by labeling LC3. VMP1, as an autophagy-related gene that has been identified, can also participate in the autophagy process of cells. So, the mRNA and protein expression levels of LC3 and Vmp1 after transfection with si-UCA1 were detected by qRTPCR and Western blot analyses. Compared with control and siRNA-NC cells, the expression of LC3 and VMP1 mRNA (Figures 3A, B; Table 5) and protein (Figures 3C-E; Table 5) in si-UCA1 cells was significantly increased $(\mathrm{P}<0.05)$. These results indicated that IncRNA UCA1 may at least partially activate the signaling pathway directly and then regulate the expression of LC3/VMP1 to promote cell proliferation, migration and invasion.

\section{DISCUSSION}

As a gynecological disorder, endometriosis is the disease which the main clinical complications include severe menstrual and nonmenstrual pain and subfertility (14). The most common hypothesis about the etiology of EMS is retrograde menstruation. However, implant or persistent deposition of lesions in the pelvic cavity is another reason that can be influenced by inflammatory, hormonal and immunologic environments (1). The diagnosis of endometriosis is inadequate and usually delayed. The incubation period from the onset of symptoms to a clear diagnosis is longer. Among patients 18-45 years old, the average delay time is 6.7 years, and some patients require surgical diagnosis (15). Endometriosis has biological characteristics similar to those of malignant tumors with a variety of pathological morphologies and invasion, which seriously affects the patient's quality of life. Therefore, it is of great significance to study the pathogenesis of endometriosis, which may contribute to the prevention, diagnosis, treatment and improvement of the prognosis of the disease.

It is estimated that $70 \%$ to $90 \%$ of transcription results in long noncoding RNAs in mammalian genomes (16). In the epigenetic state of the human genome, long noncoding RNAs are important

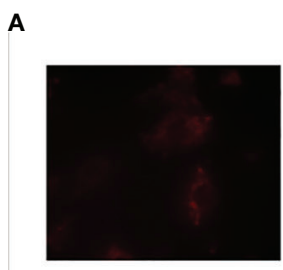

Control

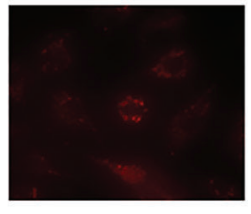

siRNA-NC

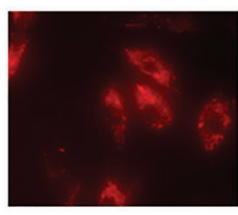

si-UCA1
B

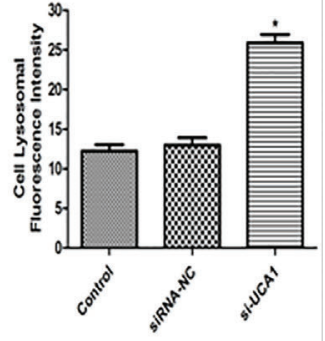

D

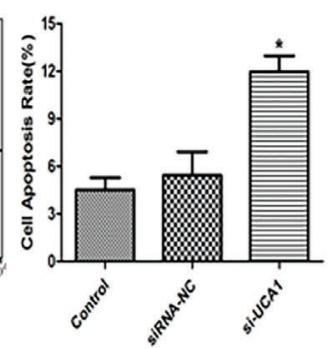

FIGURE 2 | Effect of UCA1 on ESC autophagy and apoptosis in vitro. (A, B) In the cell lysosomal fluorescence intensity assay, the cell lysosomal fluorescence intensity was significantly increased after transfection with si-UCA1 compared to the siRNA-NC and control groups (P*<0.05). (C, D) In the cell apoptosis assay, the apoptosis ability of cells was significantly increased with si-UCA1 transfection compared to the control and siRNA-NC groups ( ${ }^{*} \mathrm{P}<0.05$ ). 


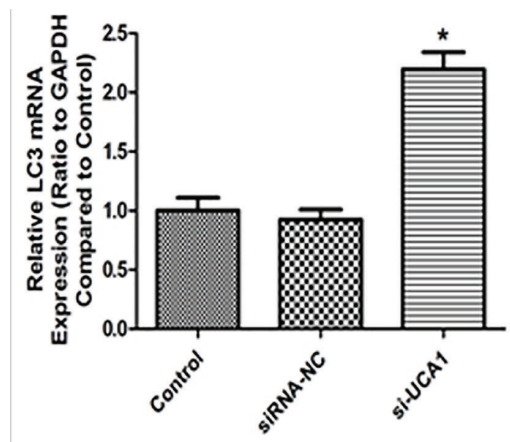

C

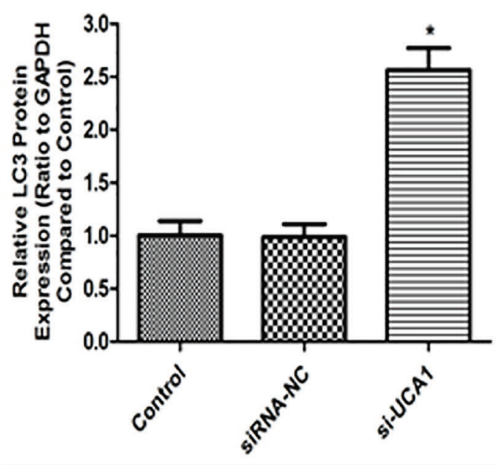

B

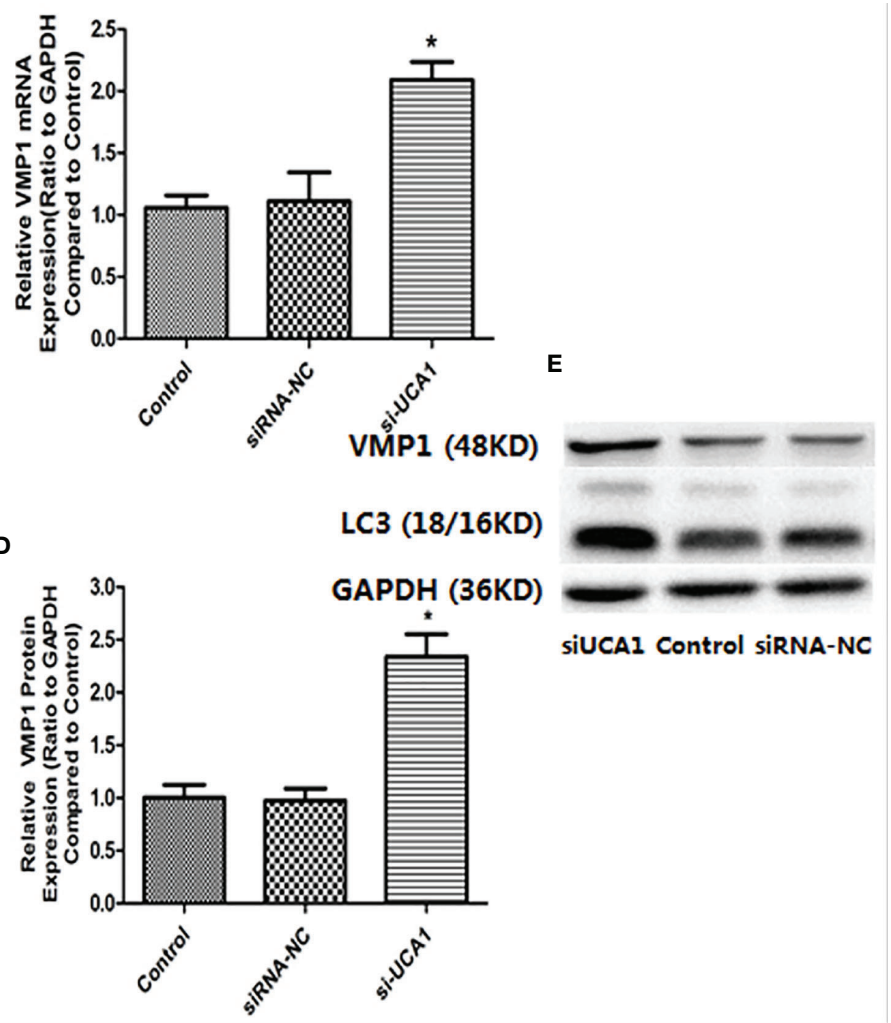

FIGURE 3 | (A, B) In the si-UCA1 group, LC3 and VMP1 mRNA levels were significantly increased compared with those in the control and siRNA-NC groups $(\mathrm{P}<0.05)$. (C, D) Compared to the control and siRNA-NC groups, LC3 and VMP1 protein expression levels were significantly increased in cells transfected with si-UCA1 (P<0.05). (E) LC3 and VMP1 protein levels after si-UCA1 transfection.

TABLE 5 | LC3 and VMP1 mRNA and protein levels in si-UCA1-transfected cells compared to the control and siRNA-NC groups.

\begin{tabular}{lccccc}
\hline Target gene & \multicolumn{2}{c}{ Relative expression level compared to Control } & & P Value(Control vs. siRNA-NC) & P Value(Control vs. si-UCA1) \\
\cline { 2 - 4 } & Control & siRNA-NC & si-UCA1 & & 0.5924 \\
\hline LC3 mRNA & $1.00 \pm 0.11$ & $0.92 \pm 0.08$ & $2.20 \pm 0.14$ & 0.9438 & $0.001^{*}$ \\
LC3 protein & $1.00 \pm 0.14$ & $0.99 \pm 0.12$ & $2.57 \pm 0.21$ & 0.8418 & $0.001^{*}$ \\
VMP1 mRNA & $1.00 \pm 0.10$ & $1.11 \pm 0.23$ & $2.09 \pm 0.15$ & 0.8773 & $0.001^{*}$ \\
VMP1 protein & $1.00 \pm 0.12$ & $0.97 \pm 0.11$ & $2.34 \pm 0.21$ & $0.001^{*}$ \\
\hline
\end{tabular}

regulators. In addition to their involvement in normal physiology, the expression and function of lncRNAs have been proven to be related to many diseases, including cancer (17). The occurrence of endometriosis has similar clinical characteristics to malignant tumors. Whether the pathogenesis is similar to that of malignant tumors has become a research direction of endometriosis etiology. A total of 1277 lncRNAs (789 downregulated and 488 upregulated) and 1216 mRNAs (638 downregulated and 578 upregulated) between eutopic and normal endometrium were differentially expressed by microarray expression profiling. Differentially expressed lncRNAs have been found to be associated with immune regulation and the cell cycle by gene ontology analysis and pathway analysis (18). As a types of lncRNA, UCA1, which is located on human chromosome 19p13.12, has been widely confirmed in a variety of tumors due to its carcinogenic effect (19). C/EBP $\alpha$ and Ets-2 are the transcription factors which can regulate the expression of UCA1, and the cancer progression can be promoted by UCA1 overexpression through different pathways, including PI3K, AKT, and mTOR-STAT3 signal pathways (20-22). UCA1 can also interact with microRNAs (miRNAs), a type of regulatory ncRNA to compete endogenous RNA in cancer cells. UCA1 promotes the development of tumors by regulating cell proliferation (23-25), invasion $(24,25)$, migration (25), metastasis (26), apoptosis (27), metabolism (22), survival (24), radiosensitivity and 
chemoresistance $(28,29)$. Thus, we have focused on whether UCA1 has significance in the study of endometriosis.

In this study, qRT-PCR analysis showed that the expression level of UCA1 in the eutopic endometrium of patients with endometriosis was significantly higher than that in the normal endometrium. The results demonstrated that high expression of UCA1 might accelerate the progression of EMS, which is consistent with its changes in various malignant tumors. Our results suggested that UCA1 might be involved in the occurrence and development of EMS by promoting cell proliferation and inhibiting apoptosis.

As a disease with growth characteristics, cell proliferation and apoptosis play important and indispensable roles in the development of EMS. For normal development and tissue size homeostasis, the coordination and balance between cell proliferation and apoptosis are crucial (30) as disease occurs once the balance is broken. Apoptosis is a unique form of programmed cell death defined by characteristic morphological and biochemical events that can lead to the effective removal of cells from tissues without causing inflammation (31). Apoptosis is a rational and positive process that can fight the defense mechanism of pathogens and is essential for embryogenesis and cell homeostasis. Previous studies have indicated that apoptosis indices of eutopic endometrium in patients with EMS are lower than those in women without EMS (32). Endometrial cells are fundamentally different in women with and without EMS, which has been verified by increasing evidence. The proliferation of endometrial cells in patients with EMS is enhanced, and the implantation ability and survival in ectopic sites are increased. The decreased sensitivity of endometrial tissue to spontaneous apoptosis leads to abnormal implantation and growth of ectopic endometrium. Apoptosis may play an important role in the development of EMS (33).

Autophagy is a closely coordinated process that delivers cellular material to lysosomes for degradation and plays an important role in maintaining cell homeostasis (34). New research has shown that autophagy is closely related to EMS. However, autophagy may be upregulated or downregulated in EMS through different signaling pathways. Thus, it remains unknown if autophagy is a foe or friend in EMS (35). Further research on the exact role of autophagy in EMS, especially in vivo studies and the detection of more autophagy-related proteins, has become particularly important.

Several autophagy-related genes (ATGs), including microtubule-associated light chain 3 (LC3) and beclin-1, play important roles in autophagy and are often regarded as potential biomarkers of autophagy (36). In different stages of autophagy, Beclin-1 and LC3 play a role. Although Beclin-1 is considered to be an important part of autophagy initiation, LC3 is involved in the later stages, and LC3-II is a marker of autophagy (36). A previous study has reported that both LC3 and LysoTracker are upregulated in different biological events during autophagy (37).

Specific genes known as ATGs regulate autophagy. Many ATG proteins, including vacuole membrane protein 1 (Vmp1), have been identified. VMP1 interacts with Beclin-1, which positively regulates the formation of autophagosomes (38).
In addition, it is known that autophagy can inhibit apoptosis and that apoptosis can regulate autophagy, but the detailed mechanism of these two pathways is still not clear in ESCs (39).

We can use high-throughput microarray to identify and screen specific lncRNAs, the new markers of some cancers. RNA interference mediated selective silencing of lncRNA may be a therapeutic option for some cancers. In this study, we found that the expression of UCA1 in the eutopic endometrium of patients with endometriosis was higher than that in the normal endometrium by qRT-PCR. In vivo, the expression levels of UCA1 in ESCs and the proliferation abilities of cells decreased significantly after knockout of lncRNA UCA1. The results show that UCA1 may promote cell proliferation of EMS. After si-UCA1 transfection, the apoptosis ability of ESCs was significantly enhanced by flow cytometry. LC3 is a marker protein on the autophagosome membrane. Since LC3 is needed during the formation of the autophagosome membrane, the autophagosome can be identified by labeling LC3. VMP1, as an autophagy-related gene that has been identified, can also participate in the autophagy process of cells. So we detected the mRNA and protein levels of LC3 and VMP1 and found that were significantly increased after UCA1 knockout, which further demonstrated that UCA1 may inhibit the autophagy of ESCs. These results indicated that UCA1 has a significant effect on the proliferation, apoptosis and autophagy of ESCs. The strong proliferation ability and the decreased autophagy and apoptosis increase the ectopic survival ability of endometrial cells and cause the occurrence of EMS. The role of UCA1 in the pathogenesis of endometriosis needs further study.

In conclusion, lncRNA-UCA1 is highly expressed in the eutopic endometrium of patients with endometriosis. Knockout of the UCA1 gene inhibits the proliferation of ESCs, promotes apoptosis, promotes autophagy and participates in the occurrence and development of endometriosis. Future animal research and bioinformatics evaluation will help to determine the mechanism of UCA1 in endometriosis.

\section{DATA AVAILABILITY STATEMENT}

The original contributions presented in the study are included in the article, further inquiries can be directed to the corresponding author/s.

\section{ETHICS STATEMENT}

The studies involving human participants were reviewed and approved by Medical Ethics Committee of Shengjing Hospital Affiliated to China Medical University. The patients/participants provided their written informed consent to participate in this study. 


\section{AUTHOR CONTRIBUTIONS}

LJ conducted main part of the experiment and was the major contributor in writing the manuscript. YW was responsible for part of the literature search and experiment. ZF and DL collected specimens and took the images. LO provided topic support and experiment consultation. YL was responsible for drawing and reviewing pictures. KL was responsible for checking the data, reviewing, and revising the article. All authors contributed to the article and approved the submitted version.

\section{FUNDING}

This research received the support from "Scientific research funding project of Liaoning Provincial Department of Science

\section{REFERENCES}

1. Parasar P, Ozcan P, Terry KL. Endometriosis: Epidemiology, Diagnosis and Clinical Management. Curr Obstet Gynecol Rep (2017) 6(1):34-41. doi: 10.1007/s13669-017-0187-1

2. Sampson JA. Metastatic or Embolic Endometriosis, due to the Menstrual Dissemination of Endometrial Tissue into the Venous Circulation. Am J Pathol (1927) 3(2):93-110.43.

3. Hernandes C, Silveira P, Rodrigues Sereia AF, Christoff AP, Mendes H, Valter de Oliveira LF, et al. Microbiome Profile of Deep Endometriosis Patients: Comparison of Vaginal Fluid, Endometrium and Lesion. Diagnostics (Basel) (2020) 10(3):163. doi: 10.3390/diagnostics10030163

4. Min L, Garbutt C, Tu C, Hornicek F, Duan Z. Potentials of Long Noncoding RNAs (LncRNAs) in Sarcoma: From Biomarkers to Therapeutic Targets. Int J Mol Sci (2017) 18(4):731. doi: 10.3390/ijms18040731

5. Wang XS, Zhang Z, Wang HC, Cai JL, Xu QW, Li MQ, et al. Rapid Identification of UCAlas a Very Sensitive and Specific Unique Marker for Human Bladder Carcinoma. Clin Cancer Res (2006) 12(16):4851-8. doi: 10.1158/1078-0432.CCR-06-0134

6. Nie W, Ge HJ, Yang XQ, Sun X, Huang H, Tao X, et al. LncRNA-UCA1 exerts oncogenic functions in non-small cell lung cancer by targeting miR-193a-3p. Cancer Lett (2016) 371(1):99-106. doi: 10.1016/j.canlet.2015.11.024

7. Zuo ZK, Gong Y, Chen XH, Ye F, Yin ZM, Gong QN, et al. TGFß1-Induced LncRNA UCA1 Upregulation Promotes Gastric Cancer Invasion and Migration. DNA Cell Biol (2017) 36(2):159-67. doi: 10.1089/dna.2016.3553

8. Wang F, Zhou J, Xie X, Hu J, Chen L, Hu Q, et al. Involvement of SRPK1 in cisplatin resistance related to long non-coding RNA UCA1 in human ovarian cancer cells. Neoplasma (2015) 62(3):432-8. doi: 10.4149/neo_2015_051

9. Bian Z, Jin L, Zhang J, Yin Y, Quan C, Hu Y, et al. LncRNA-UCA1 enhances cell proliferation and 5-fluorouracil resistance in colorectal cancer by inhibiting miR-204-5p. Sci Rep (2016) 6:23892. doi: 10.1038/srep23892

10. Khalaj K, Miller JE, Lingegowda H, Fazleabas AT, Young SL, Lessey BA, et al. Extracellular vesicles from endometriosis patients are characterized by a unique miRNA-lncRNA signature. JCI Insight (2019) 4(18):e128846. doi: $10.1172 /$ jci.insight. 128846

11. Yu J, Chen LH, Zhang B, Zheng QM. The modulation of endometriosis by IncRNA MALAT1 via NF-kB/iNOS. Eur Rev Med Pharmacol Sci (2019) 23 (10):4073-80. doi: 10.26355/eurrev_201905_17908

12. Zhu MB, Chen LP, Hu M, Shi Z, Liu YN. Effects of lncRNA BANCR on endometriosis through ERK/MAPK pathway. Eur Rev Med Pharmacol Sci (2019) 23(16):6806-12. doi: 10.26355/eurrev_201908_18719

13. Wang D, Wang D, Wang N, Long Z, Ren X. Long Non-Coding RNA BANCR Promotes Endometrial Cancer Cell Proliferation and Invasion by Regulating MMP2 and MMP1 via ERK/MAPK Signaling Pathway. Cell Physiol Biochem (2016) 40(3-4):644-56. doi: 10.1159/000452577

14. Tapmeier TT, Nazri HM, Subramaniam KS, Manek S, Garbutt K, Flint EJ, et al. Protocol for a longitudinal, prospective cohort study investigating the and Technology (No.2020JH2/10300050)", "Research funding project of Liaoning Provincial Department of Education (No.JC2019012)", and "Shenyang Science and Technology Planning Project (No.19-112-4-020)".

\section{ACKNOWLEDGMENTS}

The authors thank the support of the funding "Scientific research funding project of Liaoning Provincial Department of Science and Technology (No.2020JH2/10300050)", "Research funding project of Liaoning Provincial Department of Education (No.JC2019012)", and "Shenyang Science and Technology Plan Project (No.19-112-4-020)" to this article.

biology of uterine fibroids and endometriosis, and patients' quality of life: the FENOX study. BMJ Open (2020) 10(3):e032220. doi: 10.1136/bmjopen-2019032220

15. Nnoaham KE, Hummelshoj L, Webster P, d'Hooghe T, de Cicco Nardone F, de Cicco Nardone C, et al. Impact of endometriosis on quality of life and work productivity: a multicenter study across ten countries. Fertil Steril (2011) 96 (2):366-73.e8. doi: 10.1016/j.fertnstert.2011.05.090

16. Lee JT. Epigenetic Regulation by Long Noncoding RNAs. Science (2012) 338 (6113):1435-9. doi: 10.1126/science.1231776

17. Morlando M, Fatica A. Alteration of Epigenetic Regulation by Long Noncoding RNAs in Cancer. Int J Mol Sci (2018) 19(2):570. doi: 10.3390/ijms19020570

18. Wang Y, Li Y, Yang Z, Liu K, Wang D. Genome-Wide Microarray Analysis of Long Non-Coding RNAs in Eutopic Secretory Endometrium with Endometriosis. Cell Physiol Biochem (2015) 37(6):2231-45. doi: 10.1159/ 000438579

19. Fang Z, Zhao J, Xie W, Sun Q, Wang H, Qiao B. LncRNA UCA1 promotes proliferation and cisplatin resistance of oral squamous cell carcinoma by sunppressing miR-184 expression. Cancer Med (2017) 6(12):2897-908. doi: $10.1002 /$ cam 4.1253

20. Wu W, Zhang S, Li X, Xue M, Cao S, Chen W. Ets-2 regulates cell apoptosis via the Akt pathway, through the regulation of urothelial cancer associated 1, a long non-coding RNA, in bladder cancer cells. PLoS One (2013) 8(9):e73920. doi: 10.1371/journal.pone.0073920

21. Hughes JM, Legnini I, Salvatori B, Masciarelli S, Marchioni M, Fazi F, et al. $\mathrm{C} / \mathrm{EBP} \alpha-\mathrm{p} 30$ protein induces expression of the oncogenic long non-coding RNA UCA1 in acute myeloid leukemia. Oncotarget (2015) 6(21):18534-44. doi: 10.18632/oncotarget.4069

22. Li Z, Li X, Wu S, Xue M, Chen W. Long non-coding RNA UCA1 promotes glycolysis by upregulating hexokinase 2 through the mTOR-STAT3/ microRNA143 pathway. Cancer Sci (2014) 105(8):951-5. doi: 10.1111/cas.12461

23. Wang ZQ, Cai Q, Hu L, He CY, Li JF, Quan ZW, et al. Long noncoding RNA UCA1 induced by SP1 promotes cell proliferation via recruiting EZH2 and activating AKT pathway in gastric cancer. Cell Death Dis (2017) 8(6):e2839. doi: 10.1038/cddis.2017.143

24. Qian Y, Liu D, Cao S, Tao Y, Wei D, Li W, et al. Upregulation of the long noncoding RNA UCA1 affects the proliferation, invasion, and survival of hypopharyngeal carcinoma. Mol Cancer (2017) 16(1):68. doi: 10.1186/s12943017-0635-6

25. Xue M, Chen W, Li X. Urothelial cancer associated 1: a long noncoding RNA with a crucial role in cancer. J Cancer Res Clin Oncol (2016) 142(7):1407-19. doi: 10.1007/s00432-015-2042-y

26. Wang ZQ, He CY, Hu L, Shi HP, Li JF, Gu QL, et al. Long noncoding RNA UCA1 promotes tumour metastasis by inducing GRK2 degradation in gastric cancer. Cancer Lett (2017) 408:10-21. doi: 10.1016/j.canlet.2017.08.013

27. Yu SY, Dong B, Zhou SH, Tang L. LncRNA UCA1 modulates cardiomyocyte apoptosis by targeting miR-143 in myocardial ischemia-reperfusion injury. Int J Cardiol (2017) 247:31. doi: 10.1016/j.ijcard.2017.05.055 
28. Fotouhi Ghiam A, Taeb S, Huang X, Huang V, Ray J, Scarcello S, et al. Long non-coding RNA urothelial carcinoma associated 1 (UCA1) mediates radiation response in prostate cancer. Oncotarget (2017) 8(3):4668-89. doi: 10.18632/oncotarget.13576

29. Fan Y, Shen B, Tan M, Mu X, Qin Y, Zhang F, et al. Long non-coding RNA UCA1 increases chemoresistance of bladder cancer cells by regulating Wnt signaling. FEBS J (2014) 281(7):1750-8. doi: 10.1111/febs.12737

30. Song Y, Fu J, Zhou M, Xiao L, Feng X, Chen H, et al. Activated Hippo/YesAssociated Protein Pathway Promotes Cell Proliferation and Anti-apoptosis in Endometrial Stromal Cells of Endometriosis. J Clin Endocrinol Metab (2016) 101(4):1552-61. doi: 10.1210/jc.2016-1120

31. Kerr JF, Wyllie AH, Currie AR. Apoptosis: a basic biological phenomenon with wide-ranging implications in tissue kinetics. Br J Cancer (1972) 26 (4):239-57. doi: 10.1038/bjc.1972.33

32. Szymanowski K. Apoptosis pattern in human endometrium in women with pelvic endometriosis. Eur J Obstet Gynecol Reprod Biol (2007) 132(1):107-10. doi: 10.1016/j.ejogrb.2006.04.008

33. Harada T, Kaponis A, Iwabe T, Taniguchi F, Makrydimas G, Sofikitis N, et al. Apoptosis in human endometrium and endometriosis. Hum Reprod Update (2004) 10(1):29-38. doi: 10.1093/humupd/dmh007

34. Onorati AV, Dyczynski M, Ojha R, Amaravadi RK. Targeting autophagy in cancer. Cancer (2018) 124(16):3307-18. doi: 10.1002/cncr.31335

35. Zhan L, Li J, Wei B. Autophagy in endometriosis: Friend or foe? Biochem Biophys Res Commun (2018) 495(1):60-3. doi: 10.1016/j.bbrc.2017.10.145
36. Ozpolat B, Benbrook DM. Targeting autophagy in cancer management strategies and developments. Cancer Manag Res (2015) 7:291-9. doi: 10.2147/ CMAR.S34859

37. Chikte S, Panchal N, Warnes G. Use of LysoTracker dyes: a flow cytometric study of autophagy. Cytometry A (2014) 85(2):169-78. doi: 10.1002/ cyto.a. 22312

38. Dusetti NJ, Jiang Y, Vaccaro MI, Tomasini R, Azizi Samir A, Calvo EL, et al. Cloning and expression of the rat vacuole membrane protein 1 (VMP1), a new gene activated in pancreas with acute pancreatitis, which promotes vacuole formation. Biochem Biophys Res Commun (2002) 290(2):641-9. doi: 10.1006/ bbrc.2001.6244

39. Fan YJ, Zong WX. The cellular decision between apoptosis and autophagy. Chin J Cancer (2013) 32(3):121-9. doi: 10.5732/cjc.012.10106

Conflict of Interest: The authors declare that the research was conducted in the absence of any commercial or financial relationships that could be construed as a potential conflict of interest.

Copyright (c) 2021 Jiang, Wan, Feng, Liu, Ouyang, Li and Liu. This is an open-access article distributed under the terms of the Creative Commons Attribution License (CC BY). The use, distribution or reproduction in other forums is permitted, provided the original author(s) and the copyright owner(s) are credited and that the original publication in this journal is cited, in accordance with accepted academic practice. No use, distribution or reproduction is permitted which does not comply with these terms. 